\title{
Pharmacokinetics and dose requirements of factor VIII over the age range $3-74$ years
}

\author{
A population analysis based on 50 patients with long-term prophylactic treatment \\ for haemophilia $A$
}

\author{
Sven Björkman • Anna Folkesson • Siv Jönsson
}

Received: 4 March 2009/Accepted: 27 May 2009/Published online: 26 June 2009

(C) Springer-Verlag 2009

\begin{abstract}
Purpose The three aims of this investigation were (1) to develop a population pharmacokinetic (PK) model for factor VIII (FVIII) in haemophilia A patients, with estimates of inter-occasion and inter-individual variance, (2) to investigate whether appropriate dosing of FVIII for regular prophylaxis can be calculated according to patient characteristics, and (3) to present dosing recommendations for initiating prophylactic treatment.

Methods A population PK model was developed using data from four PK studies on patients aged 7-74 years. The model was tested on sparse FVIII data from 42 outpatient visits by haemophilia prophylaxis patients aged 3-66 years. Dose requirements for prophylaxis were calculated both according to the population model and from empirical Bayesian estimates of FVIII PK in the individual patients. Results The study data were well characterised by a twocompartment PK model. Body weight, age and type of FVIII preparation (plasma-derived or recombinant) were identified as significant covariates. Inter-occasion variance was lower than inter-individual variance for both clearance and volume of the central compartment. The model could
\end{abstract}

S. Björkman $(\bowtie) \cdot S$. Jönsson

Department of Pharmaceutical Biosciences, Uppsala University,

Box 591,

SE-751 24 Uppsala, Sweden

e-mail: sven.bjorkman@farmbio.uu.se

\section{A. Folkesson}

Department of Pharmacology and Pharmacotherapy,

Faculty of Pharmaceutical Sciences, Copenhagen University,

Universitetsparken 2,

DK-2100 Copenhagen, Denmark

S. Jönsson

Medical Products Agency,

SE-751 03 Uppsala, Sweden reasonably predict FVIII PK in the sparse clinical data. Model-predicted doses (based on age and body weight) to maintain a recommended $0.01 \mathrm{U} / \mathrm{mL}$ trough level of FVIII with administration on alternate days started at around $60 \mathrm{U} / \mathrm{kg}$ in the small children, decreasing to $10 \mathrm{U} / \mathrm{kg}$ or less in middle age. However, "true" dose requirements, as estimated from individual PK parameter data, showed a much greater variation.

Conclusion Appropriate dosing of FVIII for prophylactic treatment cannot be calculated only from body weight and/or age. However, plausible starting doses for most patients would be $1,000 \mathrm{U}$ every other day. FVIII levels should then be checked for dose adjustment.

Keywords Factor VIII · Haemophilia A · Dosing regimens · Population pharmacokinetics · Inter-occasion variance

The treatment of haemophilia A with concentrates of the missing factor VIII (FVIII) is effective but expensive. Both clinical outcome and cost-effectiveness can however be improved by tailoring the dose according to the treatment needs and the pharmacokinetics (PK) of FVIII in the individual patient. This applies to all modes of haemophilia treatment but in particular to long-term substitution therapy, or prophylactic treatment [1-5]. Dose tailoring has so far been performed using information on the PK of FVIII in the individual patient, i.e. knowledge obtained by plasma sampling and applicable analyses. The possibility of tailoring the dose a priori based only on patient characteristics (e.g. age and weight) has not yet been investigated. Such an investigation would require first a determination of the relationships of patient characteristics with the PK of FVIII. Then, predicted dosing would have to be evaluated by comparison to true dose requirement. All this must be done for an adequate number of representative patients. In 
addition, dose tailoring based on $\mathrm{PK}$ requires that the individual PK parameters are more reliably representative for the patient than the mean parameter values in the population, i.e. that there is less variance within than between the individuals.

Relationships of FVIII PK with patient characteristics have been investigated in several studies [2, 6-14] on limited numbers of patients. Weight-adjusted clearance (CL) of FVIII (i.e. in millilitres per hour per kilogram) has generally been found to decrease with age and/or body weight (BW) during growth from infancy to adulthood, with a corresponding increase in terminal half-life $(\mathrm{t} 1 / 2)[2$, $7,10,14]$. Relationships of CL and $\mathrm{t} 1 / 2$ with plasma level of von Willebrand factor have been reported [8-11] but also refuted [12]. An increased CL and shorter t1/2 of FVIII in patients with blood group $\mathrm{O}$ [9] could not be confirmed in other studies [11, 12]. These investigations were mostly performed by two-step methodology, i.e. PK parameters were estimated separately for each subject, whereupon possible correlations with patient characteristics were examined. Population PK modelling, which combines estimation of PK parameters and correlations with patient characteristics into a single procedure [15], is a more suitable method that also allows estimation of intra- versus inter-individual variance in PK [16].

The first aim of this investigation was to develop a population PK model for FVIII in haemophilia A patients that includes applicable correlations with patient characteristics and estimates of intra-individual in comparison to inter-individual variance in the PK of FVIII. The second aim was then to investigate whether appropriate dosing of FVIII during regular prophylaxis can be calculated using the patient characteristics. The third aim was to present dosing recommendations for initiating prophylactic treatment in patients with haemophilia A.

The population PK model was developed using a set of data from 107 study occasions in 34 patients. This was compiled from three published [1, 2, 17] and one unpublished study that had been performed using regular PK study protocols. Intra-individual variance (IIV) and inter-occasion variance (IOV), i.e. between study occasions, were estimated according to a published procedure [16]. The model was then tested by application to a set of sparse data obtained in clinical practice [13, 18], collected from the medical records of 16 patients from 42 visits to the outpatient clinic. Finally, dose requirements to maintain a prophylactic $0.01 \mathrm{U} / \mathrm{mL}$ trough level of FVIII were estimated as previously described $[1,2]$ for all patients. Two methods were used and compared. "True" (or most likely) requirements were calculated from individually determined PK parameters. "Model-predicted" dosing was estimated on the basis of the population model and those patient characteristics that had been identified as significant covariates.

\section{Patients and methods}

Subjects

The study comprised 47 subjects with severe haemophilia A (defined as having an FVIII level $<1 \%$ of normal activity) and three with moderate disease (FVIII 1-5\% of normal level) from the Malmö cohort of prophylaxis patients. Data on patient characteristics, dosing and FVIII procoagulant (FVIII:C) levels vs. time were compiled from five original studies, performed between 1989 and 2004, which had all been separately approved by the Ethics Committee of Lund University. In all studies, inclusion criteria were biochemically severe or moderate disease, informed consent and no indication of circulating antibodies (inhibitors). The patients were ambulant and had no apparent bleedings at the study occasions.

The model-building data set was compiled from four original PK studies. In chronological order, the first one was a cross-over comparison (1989-1990) of the plasmaderived FVIII preparations Monoclate and Monoclate-P in 10 patients [17]. It comprised two study occasions, separated by 1 week, and yielded 317 FVIII:C values. The second study [1] explored PK-based dosing of FVIII in eight of these patients, 1-3 years later (1991-1993). It contributed a further 14 study occasions (50 FVIII:C levels). The third one [2] was a controlled study on the cost-effectiveness of PK-based dosing in 21 patients, which comprised 57 study occasions (1-4 per patient, separated by 2-9 months; 1994-1995) and contributed 221 FVIII:C values. The fourth (1996-1997; described briefly in [13]) was an investigation on the putative influence of low-dose warfarin on the disposition of FVIII:C. The patients were randomised to receive either warfarin $1.25 \mathrm{mg}$ /day orally or placebo in a cross-over fashion. Two weeks after the start of the medication, FVIII was infused over $10 \mathrm{~min}$ at a dose of $50 \mathrm{U} / \mathrm{kg}$. There was no influence of the warfarin treatment on the pharmacokinetics of FVIII:C. Consequently this treatment was disregarded in the present analysis. This investigation contributed two study occasions each for 8 patients and 126 FVIII:C values. The time between treatments was 1-2 months. Three subjects had participated in the PK-based dosing study 1.5-2.5 years earlier and another one in all four studies, over the years 1989 to 1997. In total, the number of study occasions compiled from the records was 107, the number of doses was 195 and the total number of plasma FVIII:C values was 714 (Fig. 1). The age of the patients, as recorded at each occasion, ranged from 7 to 74 years and their body weights from 26 to $124 \mathrm{~kg}$. The medians were 24 years and $68 \mathrm{~kg}$. Further demographic data are given in Table 1. The number of study occasions per patient was as follows: one ( $n=3$ patients), two $(n=7)$, three $(n=12)$, four $(n=9)$, five $(n=1)$, six $(n=1)$, and seven $(n=1)$. 


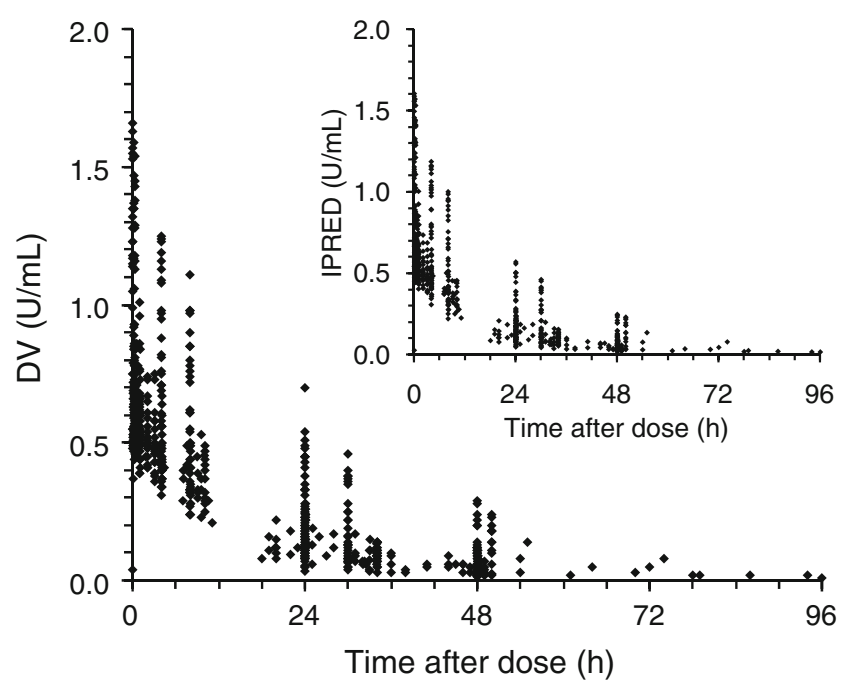

Fig. 1 The FVIII:C data $(n=714)$ used for building the population pharmacokinetic model. The inset shows individually predicted FVIII: $\mathrm{C}$ levels (IPRED)

The clinical data were collected retrospectively from medical records from the years 1997-2002 (and in a few cases prospectively in 2003-2004) [18]. The number of study occasions compiled was 42 , the number of doses was 121 and the total number of plasma FVIII:C values was 92 . The age of the patients, as recorded at each occasion, ranged from 3 to 66 years and their body weights from 17 to $78 \mathrm{~kg}$. The medians were 10 years and $37 \mathrm{~kg}$. Further demographic data are given in Table 1.
Preparations

Over the years, a number of FVIII preparations were given to the patients (Table 1). Some were successively supplied in Sweden by several manufacturers over their various periods of use. The plasma-derived FVIII concentrates were (in alphabetical order) Immunate (Immuno, Vienna, Austria/Baxter, Vienna, Austria), Monoclate and Monoclate-P (Armour, Eastbourne, UK/Urgentum, Lund, Sweden/Centeon, Stockholm, Sweden/Aventis Behring, Danderyd, Sweden) and Octonativ-M (Kabi/Pharmacia/ Pharmacia \& Upjohn/Pharmacia Plasma Products/ Biovitrum/Octapharma, all in Stockholm, Sweden). Fulllength recombinant products were Helixate (Centeon/Aventis Behring), Kogenate (Bayer, Leverkusen, Germany) and Recombinate (Baxter). A few patients received B-domaindeleted recombinant FVIII (ReFacto; Wyeth, Solna, Sweden).

All determinations of FVIII:C were performed by means of the chromogenic substrate method [19]. (The complementary one-stage assay data obtained in one study [17] were not used). All values were used as reported by the laboratory, i.e. subtraction of endogenous baseline FVIII:C levels was not attempted. The laboratory at the Department for Coagulation Disorders at Malmö University Hospital has been accredited by SWEDAC (the Swedish Board for Accreditation and Conformity Assessment) since 1993 and undergoes annual audit. The coefficient of variation (CV) for FVIII:C never exceeded $10 \%$ at the 0.25 and $1.0 \mathrm{U} / \mathrm{mL}$ levels. The $\mathrm{CV}$ determined at the time of the Monoclate
Table 1 Patient characteristics. Thirty-four patients were included in the model-building data set and 16 in the clinical data set

\footnotetext{
${ }^{a}$ Number of study occasions in which this characteristic applied ${ }^{\mathrm{b}}$ A patient may belong to more than one group
}

\begin{tabular}{|c|c|c|c|c|}
\hline \multirow[t]{2}{*}{ Characteristic } & \multicolumn{2}{|c|}{ Model-building data set } & \multicolumn{2}{|c|}{ Clinical data set } \\
\hline & Patients $(n)$ & Occasions $^{\mathrm{a}}(n)$ & Patients $(n)$ & Occasions $^{\mathrm{a}}(n)$ \\
\hline \multicolumn{5}{|l|}{ Disease severity } \\
\hline Severe $(<0.01 \quad$ U/mL FVIII:C $)$ & 31 & 97 & 16 & 42 \\
\hline Moderate & 3 & 10 & 0 & 0 \\
\hline \multicolumn{5}{|l|}{$\mathrm{Age}^{\mathrm{b}}$} \\
\hline 3-10 years (BW: 17-41 kg) & 4 & 9 & 7 & 22 \\
\hline 11-20 years (BW: 25-95 kg) & 12 & 33 & 5 & 10 \\
\hline 21-30 years (BW: 57-98 kg) & 11 & 23 & 0 & 0 \\
\hline $31-50$ years (BW: $56-124 \mathrm{~kg}$ ) & 12 & 37 & 6 & 8 \\
\hline 51-74 years (BW: 66-124 kg) & 2 & 5 & 1 & 2 \\
\hline \multicolumn{5}{|l|}{ Blood group } \\
\hline $\mathrm{O}$ & 9 & 32 & 2 & 2 \\
\hline $\mathrm{A}, \mathrm{B}$ or $\mathrm{AB}$ & 24 & 71 & 8 & 17 \\
\hline Not known & 1 & 4 & 6 & 23 \\
\hline \multicolumn{5}{|l|}{ Preparation $^{\mathrm{b}}$} \\
\hline Plasma-derived & 26 & 79 & 3 & 3 \\
\hline Full-length recombinant & 10 & 28 & 14 & 35 \\
\hline B-domain-deleted recombinant & 0 & 0 & 3 & 4 \\
\hline
\end{tabular}


study (1989) [17] was $6.2 \%$ at $0.05 \mathrm{U} / \mathrm{mL}(n=10)$ and $3.0 \%$ at $0.55 \mathrm{U} / \mathrm{mL}(n=9)$.

Pharmacokinetic modelling

The population PK modelling was carried out using nonlinear, mixed-effect modelling by means of the software NONMEM, version 6 [20]. The population PK model was built in a stepwise fashion. First, the structural model was developed in conjunction with the residual error model. Thereafter the IIV model was developed, followed by the model for IOV. Finally, covariate effects were investigated.

The structural model was either a standard one- or twocompartment PK model. The model parameters were CL and volume of distribution (V1), with inter-compartmental clearance $(\mathrm{Q})$ and volume of the second compartment (V2) added for the two-compartment model. Three different models were evaluated to describe the residual error: an additive error model, a proportional error model and a combined additive and proportional error model. Exponential models were applied to account for IIV and IOV in the PK parameters according to:

Parameter value $_{i k}=$ Typical value $\cdot \exp ^{\eta_{i}+\kappa_{i k}}$

where the subscripts $i$ and $k$ denote individual and occasion respectively and the typical value is the mean value of the parameter in the population. $\eta_{i}$ is the random effect accounting for individual deviation from the typical value (i.e. IIV) and $\kappa_{i k}$ is the random effect accounting for the IOV. $\eta_{i}$ and $\kappa_{i k}$ are assumed to be symmetrically distributed with a mean of 0 and an estimated variance of $\omega^{2}$ and $\pi^{2}$ respectively.

Allometric scaling based on body weight (BW) was applied to the PK parameters [21] as described by the equation:

Typical value $=\theta_{1} \times\left(\frac{B W}{\text { Median } B W}\right)^{\theta_{2}}$

In this expression, $\theta_{1}$ is the parameter value for a subject with a BW corresponding to the median $\mathrm{BW}$ of the population and $\theta_{2}$ is the allometric exponent. This was set to 0.75 for the clearance parameters (CL and $\mathrm{Q})$ and to 1 for the volume (V1 and V2) terms. The covariate model was then developed within NONMEM. The covariates tested (apart from BW) were age, preparation and blood group.

For fitting the model to the data, the first-order conditional estimation (FOCE) method with interaction was applied. The statistical package R, version 2.4.1 (The $\mathrm{R}$ Foundation for Statistical Computing) and Xpose version 4 [22] were used for data set checkout, exploration and model diagnostics. Model diagnostics included graphical evalua- tion, the objective function value (OFV) and the precision of parameter estimates. The main tool for selection between hierarchical models was the likelihood ratio test, i.e. the difference in OFV between models. OFV, which is a measurement of goodness of fit, is proportional to minus two times the logarithm of the likelihood $(-2 \log$ likelihood) of the data and the difference in OFV for two models is approximately $\chi^{2}$ distributed. If the models differ by one parameter, a difference in OFV of greater than 3.84 is significant at the $5 \%$ level. Corresponding values for $P=0.01$ and $P=0.001$ are 6.63 and 10.83 respectively.

The covariate model was built using stepwise forward inclusion of selected relations followed by backward elimination [23, 24]. In the forward inclusion, all selected covariate-parameter relations were included into the model separately. The most significant model (i.e. the model with greatest reduction in OFV) was retained in the next step, and all of the remaining covariate-parameter relations were included again. The full model was defined when no more covariate-parameter relations could be added on the basis of the statistical criterion $(P=0.05)$. From the full model, each parameter-covariate relationship was left out of the model one at a time (backward elimination) and the relation was kept in (or dropped from) the model using the stricter criterion $(P=0.01)$. The final model was obtained when all covariate-parameter relations were significant according to this criterion.

Individual values for $\mathrm{CL}, \mathrm{Q}, \mathrm{V} 1, \mathrm{~V} 2$ and elimination $\mathrm{t} 1 / 2$ were obtained for all patients and all study occasions as empirical Bayes estimates (EBE), i.e. by Bayesian estimation using the population model parameters in conjunction with the FVIII:C data from each individual [25]. Shrinkage of EBEs of the inter-individual random effect toward the population mean, i.e. $\eta$-shrinkage $\left(S h r_{\eta}\right)$, was estimated as $[26,27]$ :

$S h r_{\eta}=1-\frac{S D\left(\eta_{E B E}\right)}{\omega}$

where $\mathrm{SD}\left(\eta_{\mathrm{EBE}}\right)$ is the standard deviation of EBEs of $\eta$ and $\omega$ is the population model estimate of the standard deviation in $\eta$. To assess the relevance of diagnostic graphs involving individual predictions (IPRED), $\varepsilon$-shrinkage $\left(S h r_{\varepsilon}\right)$ was calculated as $[26,27]$ :

$S h r_{\varepsilon}=1-S D($ IWRES $)$

where IWRES are the individual weighted residuals.

The final model was applied to the clinical data set. Population predictions of FVIII:C levels (PRED) and PK parameters were obtained using the typical model parameters and the actual values of the relevant covariates. In addition, corresponding individual predicted values (IPRED) and PK parameters were obtained by means of Bayesian estimation. 
The accuracy and precision with which the model predicted the FVIII:C versus time data in the clinical set were estimated as the prediction errors $(P E)$ and the absolute prediction errors $(A P E)$ :

$P E=\frac{P R E D-D V}{D V}$

$A P E=\frac{|P R E D-D V|}{D V}$

In equations 5 and $6, D V$ and PRED are the measured and the population level model-predicted FVIII:C values at the times of measurement in the data set. The corresponding calculations were also made for individual FVIII:C level predictions (IPRED).

The dose of plasma-derived FVIII needed to maintain a $0.01 \mathrm{U} / \mathrm{mL}$ trough level $\left(\mathrm{C}_{\mathrm{min}}\right)$ of exogenous FVIII:C during regular prophylactic treatment was calculated as previously described $[1,2]$ for each patient, at every study occasion, in both datasets. Dosing intervals of either 24 or $48 \mathrm{~h}$ were assumed. This was done in two ways. Modelpredicted dose requirements were calculated from population PK parameter values obtained by insertion of patient characteristics (age and weight) into the population model equation. "True" dose requirements were calculated using the empirical Bayes estimates of PK parameters. Model-predicted dose requirements were evaluated in comparison to "true" dose requirements by calculation of prediction errors (PE) according to Eq. 5, with "PRED" as the model-predicted and "DV" as the "true" dose requirement.

\section{Results}

The model development, with obtained OFV values, is described in Table 2. Only the modifications that resulted in a statistically significantly better description of the data are shown. Based on the OFV values, a two-compartment model was superior to a one-compartment model, and in both cases incorporation of an estimate of endogenous baseline FVIII:C level led to a further improvement. Incorporation of IOV for the predominant clearance and volume terms, i.e. elimination clearance (CL) and volume of the central compartment (V1), further improved the model. As for the tested covariates, body weight was accounted for by the allometric scaling. Age showed a significant influence only on (the already weight-adjusted) CL and no significant relationship with (weight-adjusted) V1. Patients with blood group $\mathrm{O}$ showed a trend towards a higher $\mathrm{CL}$, however this was not retained as a significant covariate. Type of preparation had a significant influence on both CL and $\mathrm{V} 1$, however this could be ascertained only for full-length recombinant products and not for B-domain-deleted FVIII (which was not used in the protocol studies, Table 1).

Thus, the final population PK model for FVIII was a two-compartment model incorporating an estimate of baseline FVIII:C level. The NONMEM subroutine ADVAN3 TRANS4 was used, yielding estimates for CL, $\mathrm{V} 1, \mathrm{Q}, \mathrm{V} 2$ and elimination $\mathrm{t} 1 / 2$. A combined additive and proportional error model was applied. There was a good agreement between model predictions and observations of FVIII:C levels as demonstrated by the standard diagnostic plots (Fig. 2), whose validity is supported by the low $\varepsilon$ shrinkage, and also by visual inspection of IPRED values (Fig. 1). The primary parameter estimates are presented in

Table 2 Model-building steps that resulted in significant decreases in the objective function value (OFV)

\begin{tabular}{|c|c|c|c|}
\hline Model & & NOP & OFV \\
\hline \multicolumn{4}{|c|}{ Structural model } \\
\hline 1 & One compartment with IIV on V1 and CL & 7 & $-3,430.7$ \\
\hline 2 & One compartment with BL and IIV on V1, CL and BL & 10 & $-3,470.8$ \\
\hline 3 & Two-compartment with IIV on V1 and CL & 9 & $-3,462.4$ \\
\hline 4 & Two-compartment with BL and IIV on V1, CL and BL & 12 & $-3,486.9$ \\
\hline \multicolumn{4}{|c|}{ Inter-occasion variance (added to model 4) } \\
\hline 5 & For V1 & 13 & $-3,592.1$ \\
\hline 6 & For $\mathrm{CL}$ & 13 & $-3,623.5$ \\
\hline 7 & For both $\mathrm{V} 1$ and $\mathrm{CL}$ & 14 & $-3,754.0$ \\
\hline \multicolumn{4}{|c|}{ Covariates (added to model 7) } \\
\hline 8 & Age as covariate on $\mathrm{CL}$ & 15 & $-3,761.6$ \\
\hline 9 & Preparation $=$ full-length recombinant, as covariate on V1 and CL (added to model 8) & 16 & $-3,781.1$ \\
\hline
\end{tabular}

$N O P$ Number of estimated parameters in the model, $I I V$ inter-individual variance, $V 1$ volume of the central compartment, $C L$ clearance, $B L$ estimation of endogenous baseline level of FVIII:C 


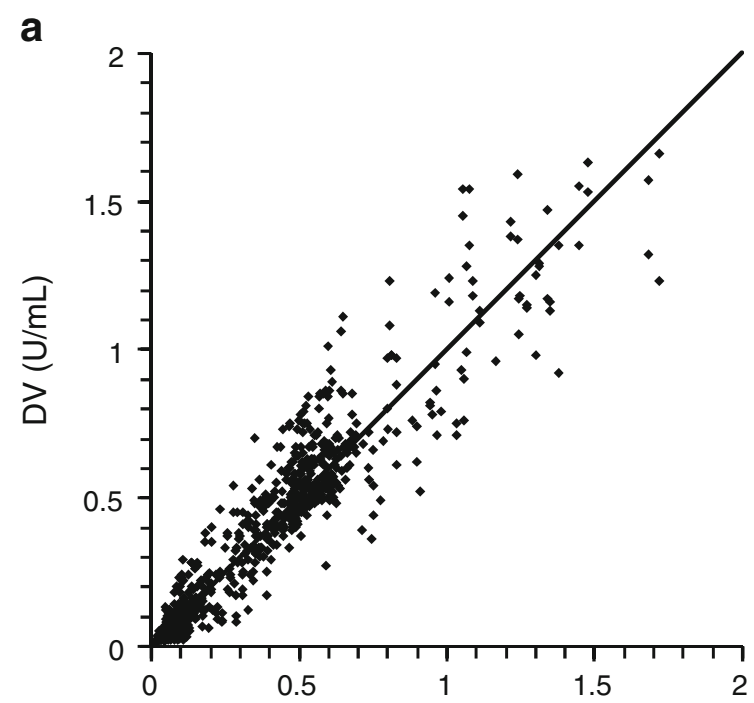

C
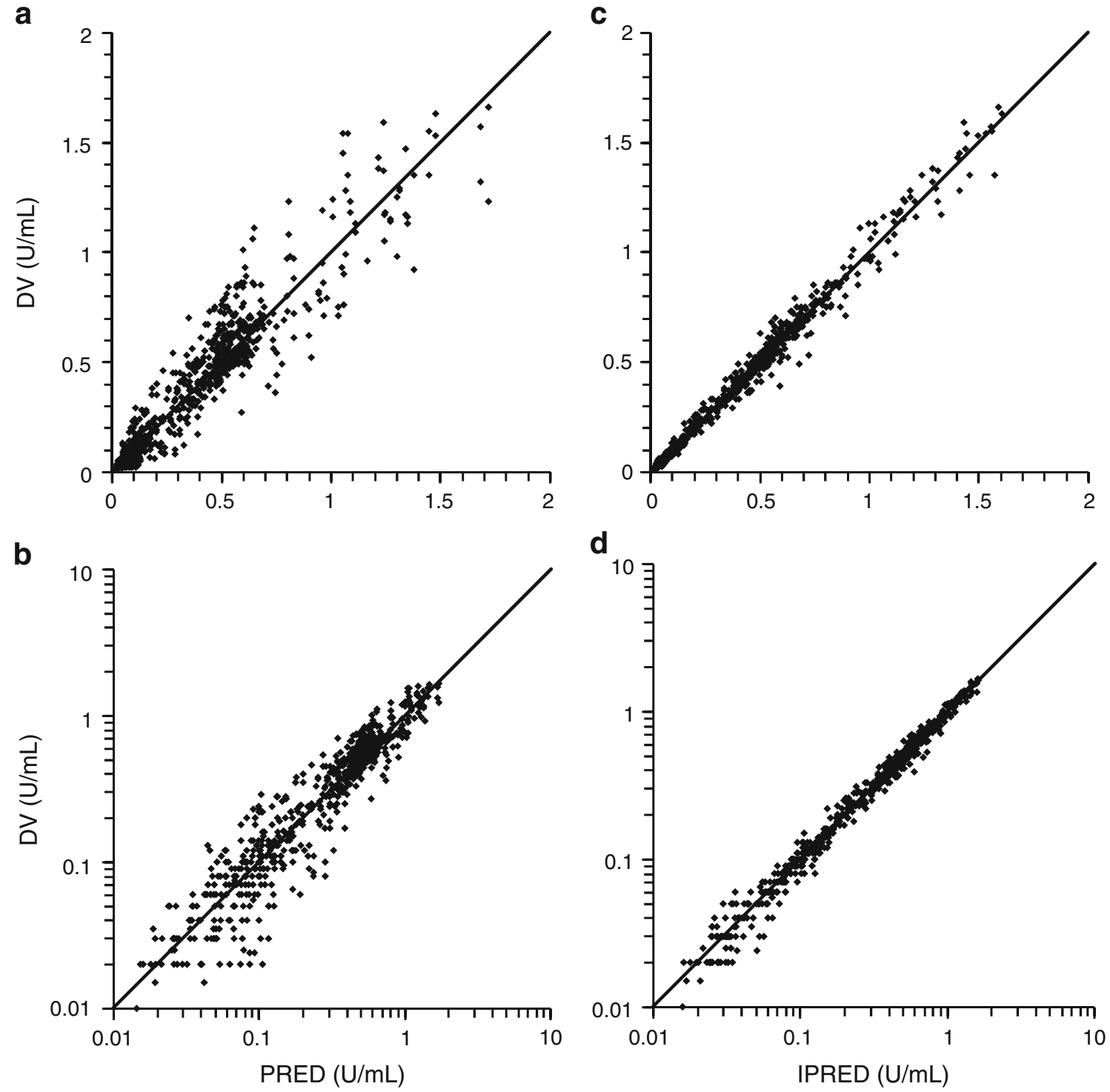

Fig. 2a-d NONMEM model diagnostic plots. a Dependent variable $(D V)$, i.e. observed FVIII:C level, versus population predicted FVIII:C level $(P R E D)$. b Logarithmic view of a. c $D V$ versus individually

Table 3. With applicable covariates, the expressions for the parameters in the model-building study group consequently were

$$
\begin{aligned}
C L(m L / h)= & 222 \times\left(\frac{B W}{68}\right)^{0.75} \\
& \times(1-0.00696 \times(\text { Age }-24))
\end{aligned}
$$

For the full-length recombinant products, this value was multiplied by $(1-0.201)$, i.e. by 0.80 .

$V 1(L)=3.52 \times\left(\frac{B W}{68}\right)$ d

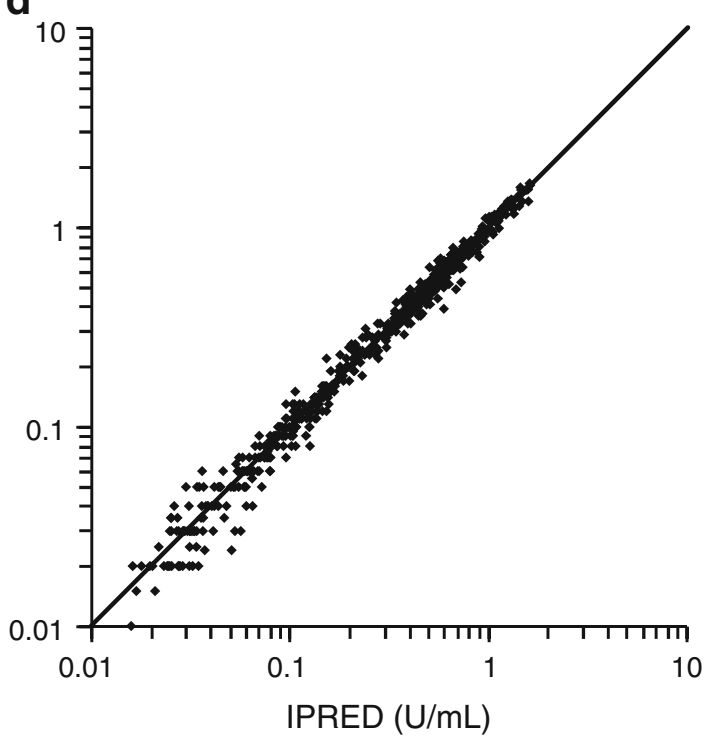

predicted FVIII:C level (IPRED). d Logarithmic view of c. Epsilon $(\varepsilon)$ shrinkage was 0.11

This equals $0.052 \mathrm{~L} / \mathrm{kg}$. For the full-length recombinant products, this value was multiplied by $(1-0.201)$, i.e. by 0.80 .

$Q(m L / h)=256 \times\left(\frac{B W}{68}\right)^{0.75}$

And:

$V 2(L)=0.241 \times\left(\frac{B W}{68}\right)$

This equals $0.0035 \mathrm{~L} / \mathrm{kg}$.

Total volume of distribution, i.e. volume of distribution at steady state $\left(\mathrm{V}_{\mathrm{ss}}\right)$ thus equalled $\mathrm{V} 1+\mathrm{V} 2=0.055 \mathrm{~L} / \mathrm{kg}$. 
$\mathrm{CL}$ and elimination $\mathrm{t} 1 / 2$ are shown as functions of age in Fig. 3. The CL value is shown for plasma-derived FVIII, after re-calculation of the estimates using the covariate factor 0.201 . For both CL and V1, IOV was considerably lower than IIV (Table 3).

For the clinical data set, the population predicted FVIII:C values (PRED) in patients $>10$ years of age had a median PE of $7.6 \%$ and a median APE of $26 \%$. The corresponding PE and APE for individual predictions (IPRED) were 3.3 and $5.5 \%$ respectively. Population model predictions tended to be poor for the children aged 3-10 years, with a median FVIII:C PE of $26 \%$ and a median APE of $31 \%$. Model-predicted CL values in these children ranged from 5.1 to $6.6 \mathrm{~mL} / \mathrm{h}$ per $\mathrm{kg} \mathrm{BW}$ and terminal half-lives from 7.3 to $9.5 \mathrm{~h}$. The median PE and APE of IPRED FVIII:C levels were 2.4 and $8.5 \%$. The individually estimated PK parameters had a wider distribution than the model predictions, with $\mathrm{CL}$ ranging from 3.1 to $8.3 \mathrm{~mL} / \mathrm{h}$ per $\mathrm{kg} \mathrm{BW}$ and terminal half-lives between 4.7 and $12 \mathrm{~h}$.

Calculated dose requirements for regular prophylaxis are shown in Fig. 4. Model-predicted doses to maintain a trough level of exogenous FVIII:C with administration every 2 days started at around $60 \mathrm{U} / \mathrm{kg}$ in the small children, decreasing to $10 \mathrm{U} / \mathrm{kg}$ or less in middle age. In terms of units needed, a 1,000-U vial would be appropriate for children, adolescents and young adults and a 500-U vial for the middle aged. "True" dose requirements, as estimated from individual PK parameter data, showed a much greater variation, however. Except for the generally decreasing trend with age, these were not well predicted by the population model. The prediction errors ranged from -95 to $+372 \%$, with a median of $+2.4 \%$ in the model-building set and $-49 \%$ in the clinical data set. With daily dosing of FVIII, model-predicted dose requirements ranged from 0.74 to $6.1 \mathrm{U} / \mathrm{kg}$, or 70 to 184 U. "True" doses required were from 39 to $668 \mathrm{U}$. The calculated "true" dose exceeded $250 \mathrm{U}$ in nine patients and $500 \mathrm{U}$ in only two patients.

\section{Discussion}

This is, to our knowledge, the first report of a population PK study of FVIII comprising the entire age range of prophylaxis patients and also the first one showing PKbased dose requirements as a function of the age of the patient. Population PK modelling has previously been applied to FVIII with the intent to determine suitable time schedules for sparse blood sampling, e.g. for studies in children [12] or for therapeutic drug monitoring [28]. In all three studies, the PK of FVIII:C was best described by a two-compartment model with a small peripheral compartment. It is still not clear whether the two-compartment characteristic reflects distribution of FVIII from the circulation or rapid initial elimination of some of the injected protein $[3,17]$.
Table 3 Parameter estimates for the final model

$\% R S E$ Relative standard error, $\% C V$ percent coefficient of variation, $S D$ standard deviation,

${ }^{\text {a }}$ Typical value for an individual with median body weight and age; i.e. $68 \mathrm{~kg}$ and 24 years

${ }^{\mathrm{b}}$ Typical value for an individual with median body weight, i.e. $68 \mathrm{~kg}$

${ }^{\mathrm{c}}$ A common value of -0.20 could be applied with marginal change in OVF $(+0.15)$

${ }^{\mathrm{d}}$ The \%RSE for corresponding variance or covariance term

\begin{tabular}{|c|c|c|}
\hline Parameter & Mean & $\% \mathrm{RSE}$ \\
\hline \multicolumn{3}{|l|}{ Structural model parameters } \\
\hline Clearance $(\mathrm{CL} ; \mathrm{mL} / \mathrm{h})^{\mathrm{a}}$ & 222 & 5.0 \\
\hline Volume of central compartment $(\mathrm{V} 1 ; \mathrm{mL})^{\mathrm{b}}$ & 3520 & 3.0 \\
\hline Inter-compartmental clearance $(\mathrm{Q} ; \mathrm{mL} / \mathrm{h})^{\mathrm{b}}$ & 256 & 30 \\
\hline Volume of peripheral compartment $(\mathrm{V} 2 ; \mathrm{mL})^{\mathrm{b}}$ & 241 & 14 \\
\hline Baseline of endogenous FVIII (BL; U/mL) & 0.012 & 17 \\
\hline \multicolumn{3}{|l|}{ Inter-individual variability parameters } \\
\hline Clearance $(\% \mathrm{CV})$ & 28 & $33^{\mathrm{d}}$ \\
\hline Volume of central compartment $(\% \mathrm{CV})$ & 17 & $37^{\mathrm{d}}$ \\
\hline Baseline $(\% \mathrm{CV})$ & 31 & $110^{\mathrm{d}}$ \\
\hline Correlation between CL and V1 & 0.64 & $44^{\mathrm{d}}$ \\
\hline \multicolumn{3}{|l|}{ Inter-occasion variability parameters } \\
\hline Clearance $(\% \mathrm{CV})$ & 13 & $31^{\mathrm{d}}$ \\
\hline Volume of central compartment $(\% \mathrm{CV})$ & 10 & $36^{\mathrm{d}}$ \\
\hline \multicolumn{3}{|l|}{ Residual variability parameters } \\
\hline Additive residual error $(\mathrm{SD} ; \mathrm{U} / \mathrm{mL})$ & 0.012 & 17 \\
\hline Proportional residual error $(\% \mathrm{CV})$ & 8.5 & 6.7 \\
\hline \multicolumn{3}{|l|}{ Covariate parameters } \\
\hline CL (\% change with age difference from 24 years) & -0.70 & 16 \\
\hline $\mathrm{CL}(\% \text { difference if preparation }=\text { full-length recombinant })^{\mathrm{c}}$ & -20 & 15 \\
\hline $\mathrm{V} 1(\% \text { difference if preparation }=\text { full-length recombinant })^{\mathrm{c}}$ & -20 & 15 \\
\hline
\end{tabular}




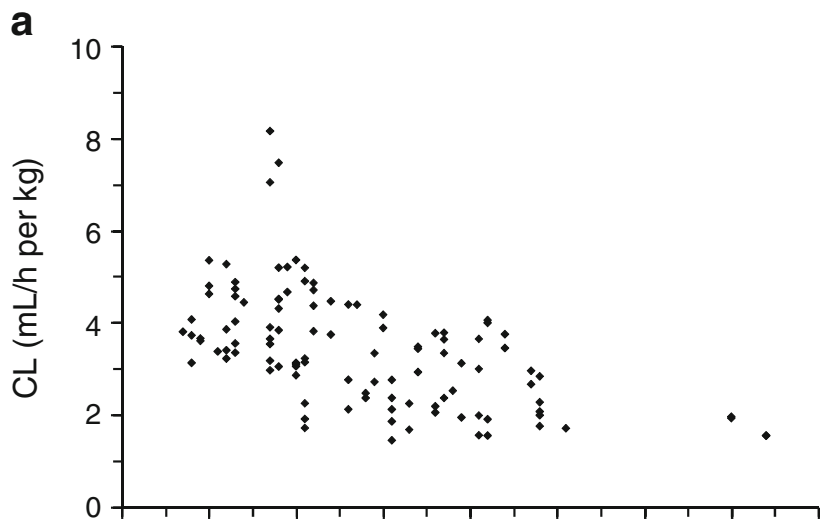

b

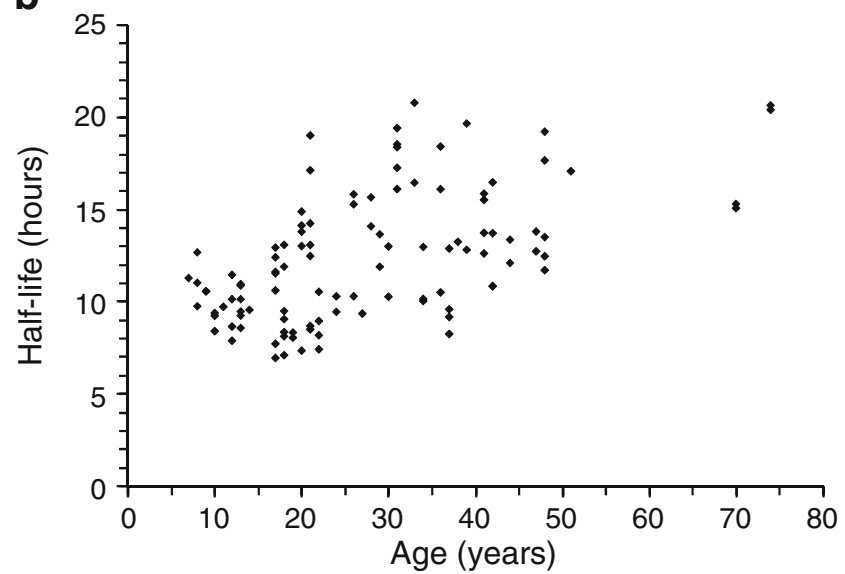

Fig. 3 a The clearance $(C L)$ of plasma-derived FVIII, normalised for total body weight, as a function of age. $\mathbf{b}$ The elimination half-life of FVIII as a function of age. Eta $(\eta)$ shrinkage was 0.04 and 0.10 respectively for the estimates of inter-individual variability of clearance and volume of the central compartment

The population mean values found for $\mathrm{CL}, \mathrm{V}_{\mathrm{ss}}$ and elimination $\mathrm{t} 1 / 2$ are in general agreement with data from conventional PK studies [3, 29]. The mean CL of $222 \mathrm{~mL} / \mathrm{h}$ in a 24-year-old, $68-\mathrm{kg}$ subject equals $3.26 \mathrm{~mL} / \mathrm{h}$ per $\mathrm{kg}$, which is similar to the accepted mean value of $3 \mathrm{~mL} / \mathrm{h}$ per $\mathrm{kg}$ in an "adult" patient, and the elimination $\mathrm{t} 1 / 2$ estimates (Fig. 3b) for "adults" are scattered around the normal mean value of $12-14 \mathrm{~h}$. The findings of a decreased CL per $\mathrm{kg}$ BW and an increased t1/2 with age are also in general agreement with previous studies $[2,7,10,14]$. The mean estimate of BL was rather high however, given that only 3 of the 50 patients were diagnosed with moderate haemophilia, and the IIV had a relative $\mathrm{CV}$ of $110 \%$. The problem that the data were not well suited for determination of BL is familiar from conventional FVIII studies $[17,29,30]$, in which "BL" is regarded more as an empirical correction factor than as an accurate estimate of endogenous FVIII:C level. More realistic estimates of BL were, however, obtained in a smaller population PK study using only "rich" data [12].

In the context of PK investigations and drug dosing, in particular when dealing with children or markedly over- weight subjects, body size can be expressed in different ways. Since total BW is often a poor descriptor, either lean body mass [12], body surface area (BSA) or allometric scaling $[21,31]$ can be applied. Scaling body weight to the power of 0.75 is similar to scaling by BSA in the weight range of the present study subjects [31]. As described in a recent review [21], using allometric body-weight scaling with the coefficients of $\mathrm{CL}$ and $\mathrm{V} 1$ fixed to 0.75 and 1 respectively confers at least two advantages in the modelling. First, experimental uncertainty in the estimation of BSA (or lean body mass) is obviated. Second, after this adjustment for body size the putative influence of other covariates can be identified. In children, age and weight are highly correlated. With allometric scaling of body weight, age remained a significant covariate only on $\mathrm{CL}$, which was predicted to decrease by $1.5 \mathrm{~mL} / \mathrm{h}$ per year of age above or
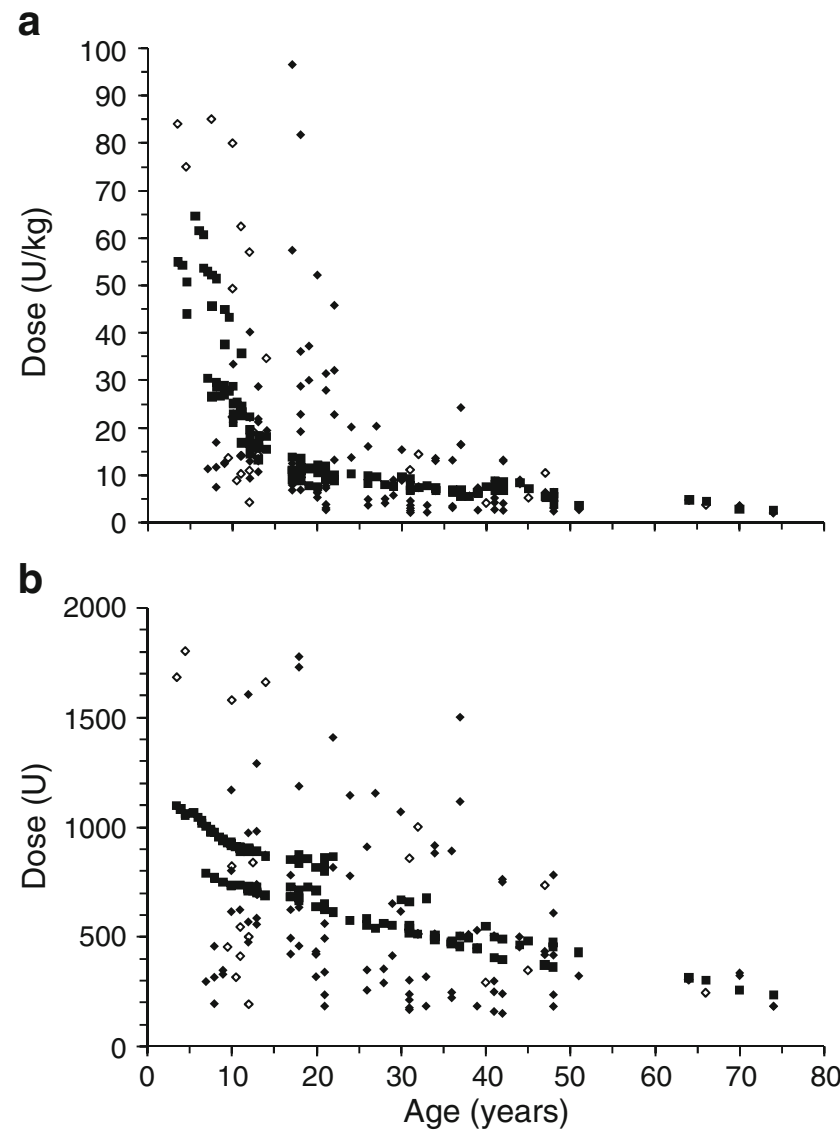

Fig. 4 Required dose of FVIII (a in units/kg bodyweight, $\mathbf{b}$ in units) to maintain a minimum level of $0.01 \mathrm{U} / \mathrm{mL}$ during continuous prophylaxis with administration every 2 days. Solid squares Model predictions (based on age and weight) for all patients (both datasets) and all occasions, solid diamonds "true" dose requirements (based on estimated individual pharmacokinetic parameter values) for all patients and occasions in the model-building data set, open diamonds "true" dose requirements for all patients and occasions in the clinical data set. In a, 5 patients (16 occasions) with true dose requirements $>100 \mathrm{U} / \mathrm{kg}$ are above the scale, and in $\mathbf{b}, 11$ patients (32 occasions) with true dose requirements $>2,000 \mathrm{U}$ 
below the median of 24 , in addition to the influence of changes in body weight. V1, on the other hand, was directly proportional to body weight over the whole age range. This agrees with a previous observation [13] that in vivo recovery $\left(\mathrm{C}_{\max }\right.$ divided by dose) of FVIII did not show any relationship to age.

Type of preparation was identified as a PK covariate. Both CL and V1 were on average 20\% lower for full-length recombinant than for plasma-derived FVIII. Both these parameter estimates are directly proportional to dose. This suggests that the discrepancies (which are in general agreement with contemporary cross-over studies [32-35]) are due to differences in the actual potency of the preparations used at the time. It was previously found [9] that the elimination $\mathrm{t} 1 / 2$ of FVIII was shorter in haemophilia patients with blood group $\mathrm{O}$ than in those with blood group $\mathrm{A}$. The effect of blood group appears to be modest, however, and it was not significant in our or in an earlier $[11,12]$ investigation.

Covariates are normally investigated in order to explain IIV. However, there is also variance in individual PK between study occasions. Hence IOV can result in biased estimates of population parameters and variance if it is not taken into account in the analysis [16]. In addition, a prerequisite for PK-based individualised dosing is that the individual variability in PK between occasions is lower than the variability between individuals. The estimated IOV was lower than the IIV for both CL and V1, which supports PK-based dose individualisation during long-term treatment with FVIII. The finding is fortunate, since individualisation of this type has been advocated and practiced for many years [1-5, 30].

The model gave poor predictions of FVIII PK for the children in the clinical data set, where Bayesian estimation based on the sparse data indicated on average unexpectedly short half-lives. The model predictions correspond better to the findings of a recent clinical study on a full-length recombinant FVIII product [14], in which children of 1-6 years of age showed a mean CL of $4.3 \mathrm{~mL} / \mathrm{h}$ per $\mathrm{kg}$ and a $\mathrm{t} 1 / 2$ that on average increased from $8.7 \mathrm{~h}$ at 1 year to $10.7 \mathrm{~h}$ at 6 years of age (compare Fig. 3). The reason for this discrepancy is not clear; the data were very sparse, however, with only two blood samples per occasion and in some cases uncertainty about the actual time of the previous (home-treatment) dose.

\section{Clinical implications}

The principle of dosing FVIII to a target trough level of $0.01 \mathrm{U} / \mathrm{mL}$ has been questioned [4, 36], however, no other treatment strategy has been shown to be superior. A correlation between time below $0.01 \mathrm{U} / \mathrm{mL}$ and risk of bleeding during prophylactic treatment has been demon- strated twice [18, 37]. FVIII is generally available in vials of 250,500 or 1,000 U. Since FVIII is expensive and transient overdosing poses no risk, the whole content of one or several vials is always given. The present study shows that FVIII cannot be dosed accurately to a certain target level only according to body weight or age. The findings suggest a suitable procedure, however. For treatment every 2 days, a plausible starting dose would be 1,000 U (or 500 $\mathrm{U}$ in the rare case of initiating prophylaxis in a middle-aged patient). The FVIII:C level should then be checked after attainment of steady state and the dose adjusted as needed. In case a too high dose of FVIII is needed with this treatment schedule, daily dosing should be considered, in which case $500 \mathrm{U}$ is nearly always adequate. Longer term clinical outcome decides whether further dose adjustment (e.g. to another trough level) is needed.

Acknowledgements We would like to thank Professor Erik Berntorp at the Centre for Thrombosis and Haemostasis at Malmö University Hospital for help and advice in the early part of this work, and nurse Karin Lindvall for help with data collection. This work was in initiated at the Hospital Pharmacy of Malmö University Hospital.

\section{References}

1. Carlsson M, Berntorp E, Björkman S, Lindvall K (1993) Pharmacokinetic dosing in prophylactic treatment of hemophilia A. Eur J Haematol 51:247-252

2. Carlsson M, Berntorp E, Björkman S, Lethagen S, Ljung R (1997) Improved cost-effectiveness by pharmacokinetic dosing of factor VIII in prophylactic treatment of haemophilia A. Haemophilia 3:96-101

3. Björkman S, Berntorp E (2001) Pharmacokinetics of coagulation factors. Clinical relevance for patients with haemophilia. Clin Pharmacokinet 40:815-832

4. Björkman S (2003) Prophylactic dosing of factor VIII and factor IX from a clinical pharmacokinetic perspective. Haemophilia 9 (Suppl. 1):101-110

5. Shapiro AD, Korth-Bradley J, Poon MC (2005) Use of pharmacokinetics in the coagulation factor treatment of patients with haemophilia. Haemophilia 11:571-582

6. Aronstam A, McLellan DS, Wassef M, Mbhata PS (1982) Effect of height and weight on the in vivo recovery of transfused factor VIII C. J Clin Pathol 35:289-291

7. Matucci M, Messori A, Donati-Cori G, Longo G, Vannini S, Morfini M, Tendi E, Rossi-Ferrini PL (1985) Kinetic evaluation of four factor VIII concentrates by model-independent methods. Scand J Haematol 34:22-28

8. Fijnvandraat K, Peters M, ten Cate JW (1995) Inter-individual variation in half-life of infused recombinant factor VIII is related to pre-infusion von Willebrand factor antigen levels. $\mathrm{Br} \mathrm{J}$ Haematol 91:474-476

9. Vlot AJ, Mauser-Bunschoten EP, Zarkova AG, Haan E, Kruitwagen CLJJ, Sixma JJ, van den Berg HM (2002) The half-life of infused factor VIII is shorter in hemophiliac patients with blood group 0 than in those with blood group A. Thromb Haemost 83:65-69

10. van Dijk K, van der Bom JG, Lenting PJ, de Groot PG, MauserBunschoten EP, Roosendaal G, Grobbee DE, van den Berg HM 
(2005) Factor VIII half-life and clinical phenotype of severe hemophilia A. Haematologica 90:494-498

11. Barnes C, Lillicrap D, Pazmino-Canizares J, Blanchette VS, Stain AM, Clark D, Hensmen C, Carcao M (2006) Pharmacokinetics of recombinant factor VIII (Kogenate-FS) in children and causes of inter-patient pharmacokinetic variability. Haemophilia 12(Suppl. 4):40-49

12. Stass $H$ (2006) Determination of minimal sampling time points for reliable pharmacokinetic evaluation of recombinant factor VIII-an exploratory population pharmacokinetic analysis in paediatric patients suffering from severe haemophilia. Haemophilia 12 (Suppl. 4):50-55

13. Björkman S, Folkesson A, Berntorp E (2007) In vivo recovery of factor VIII and factor IX: intra- and inter-individual variance in a clinical setting. Haemophilia 13:2-8

14. Blanchette VS, Shapiro AD, Liesner RJ, Hernàndez Navarro $F$, Warrier I, Schroth PC, Spotts G, Ewenstein BM (2008) Plasma and albumin-free recombinant factor VIII: pharmacokinetics, efficacy and safety in previously treated pediatric patients. J Thromb Haemost 6:1319-1326

15. Aarons L (1991) Population pharmacokinetics: theory and practice. Br J Clin Pharmacol 32:669-670

16. Karlsson MO, Sheiner LB (1993) The importance of modeling interoccasion variability in population pharmacokinetic analyses. $\mathrm{J}$ Pharmacokinet Biopharm 21:735-750

17. Björkman S, Carlsson M, Berntorp E, Stenberg P (1992) Pharmacokinetics of factor VIII in humans. Obtaining clinically relevant data from comparative studies. Clin Pharmacokinet 22:385-395

18. Ahnström J, Berntorp E, Lindvall K, Björkman S (2004) A sixyear follow-up of dosing, coagulation factor levels and bleedings in relation to joint status in the prophylactic treatment of haemophilia. Haemophilia 10:689-697

19. Rosén S (1984) Assay of factor VIII:C with a chromogenic substrate. Scand J Haematol 33(Suppl. 40):139-145

20. Beal SL, Sheiner LB (eds) (1989-1992) NONMEM users guides. Icon Development Solutions, Ellicott City, MD, USA

21. Meibohm B, Läer S, Panetta JC, Barrett JS (2005) Population pharmacokinetic studies in pediatrics: issues in design and analysis. AAPSJ 7:475-487

22. Jonsson EN, Karlsson MO (1999) Xpose-an S-PLUS based population pharmacokinetic-pharmacodynamic model building aid for NONMEM. Comput Methods Programs Biomed 58:51-64

23. Mandema JW, Verotta D, Sheiner LB (1992) Building population pharmacokinetic-pharmacodynamic models. I. Models for covariate effects. J Pharmacokinet Biopharm 20:511-528
24. Jonsson EN, Karlsson MO (1998) Automated covariate model building within NONMEM. Pharm Res 15:1463-1468

25. Sheiner LB, Beal S, Rosenberg B, Marathe VV (1979) Forecasting individual pharmacokinetics. Clin Pharmacol Ther 26:294305

26. Savic RM, Wilkins J, Karlsson MO (2006) (Un)informativeness of empirical Bayes estimate-based diagnostics. AAPS J 8(Suppl. 2). http://www.aapsj.org/abstracts/AM_2006/AAPS2006-001912.pdf. Accessed 2009-05-08

27. Karlsson MO, Savic RM (2007) Diagnosing model diagnostics. Clin Pharmacol Ther 82:17-20

28. Bolon-Larger M, Chamouard V, Bressolle F, Boulieu R (2007) A limited sampling strategy for estimating individual pharmacokinetic parameters of coagulation factor VIII in patients with hemophilia A. Ther Drug Monit 29:20-26

29. Björkman S, Carlsson M (1997) The pharmacokinetics of factor VIII and factor IX: methodology, pitfalls and applications. Haemophilia 3:1-8

30. Messori A, Longo G, Matucci M, Morfini M, Rossi Ferrini PLR (1987) Clinical pharmacokinetics of factor VIII in patients with classic haemophilia. Clin Pharmacokinet 13:365-380

31. Holford NHG (1996) A size standard for pharmacokinetics. Clin Pharmacokinet 30:329-332

32. Schwartz RS, Abildgaard CF, Aledort LM, Arkin S, Bloom AL, Brackmann HH et al (1990) Human recombinant DNA-derived antihemophilic factor (factor VIII) in the treatment of hemophilia A. New Engl J Med 323:1800-1805

33. Harrison JFM, Bloom AL, Abildgaard CF (1991) The pharmacokinetics of recombinant factor VIII. Semin Hematol 28(Suppl. 1):29-35

34. Morfini M, Longo G, Messori A, Lee M, White G, Mannucci P (1992) Pharmacokinetic properties of recombinant factor VIII compared with a monoclonally purified concentrate (Hemofil $\left.{ }^{\circledR} \mathrm{M}\right)$. Thrombos Haemostas 68:433-435

35. White GC, Courter S, Bray GL, Lee M, Gomperts ED (1997) A multicenter study of recombinant factor VIII (Recombinate ${ }^{\mathrm{TM}}$ ) in previously treated patients with hemophilia A. Thrombos Haemostas 77:660-667

36. Fischer K, Astermark J, van Der Bom JG, Ljung R, Berntorp E, Grobbee DE, van Den Berg HM (2002) Prophylactic treatment for severe haemophilia: comparison of an intermediate-dose to a highdose regimen. Haemophilia 8:753-760

37. Collins PW, Blanchette VS, Fischer K, Björkman S, Oh M, Fritsch S (2009) Break-through bleeding in relation to predicted factor VIII levels in patients receiving prophylactic treatment for severe haemophilia A. J Thrombos Hemostas 7:413-420 